\title{
Diseño y Propiedades Psicométricas del Inventario para Evaluar la Efectividad de los Equipos de Trabajo (IET)
}

\author{
Design and Psychometric Properties of the Inventory to Evaluate Work Teams \\ Effectiveness (IET)
}

\author{
Leonor Emilia Delgado-Abella ${ }^{1}$, Valeria León Rodríguez ${ }^{2}$, Angélica Viviana Pínzón Ruiz ${ }^{3}$ y \\ Alejandro Marín Contreras ${ }^{4}$
}

\begin{abstract}
Resumen
El objetivo de esta investigación fue diseñar y analizar las propiedades psicométricas del Inventario para Evaluar la Efectividad de los Equipos de Trabajo (IET). Se realizaron las siguientes fases: desarrollo de la versión preliminar del inventario con base en el modelo IMOI (Inputs-Mediators-Outputs-Inputs), validación de la prueba y análisis de sus propiedades psicométricas. La muestra total estuvo compuesta por 286 empleados de organizaciones de Bogotá, 211 mujeres (73\%) con edades comprendidas entre los 22 y los 66 años (M=33.1 y D.E.=9.9) y 75 hombres entre los 18 y los 68 años (M=32 y D.E.=11.3). El IET está formado por 46 ítems en su versión definitiva para la medida de tres dimensiones teóricas y distinguiéndose en cada dimensión los siguientes factores: en la dimensión insumos: liderazgo ( 7 ítems), trabajo en equipo (6 ítems) y, clima y cultura organizacional (5 ítems); en la dimensión mediadores: procesos de acción y transición (6 ítems) y clima de equipo (4 ítems) y, por último, en la dimensión resultados a nivel grupal y organizacional (15 ítems), y reconocimientos y recompensas (3 ítems). Se concluye que el IET posee una adecuada confiabilidad y evidencia de validez de contenido y constructo.
\end{abstract}

Palabras clave: efectividad de equipos de trabajo, equipos de trabajo, modelo IMOI, evaluación de efectividad, evaluación de efectividad de equipos de trabajo

\begin{abstract}
The objective of this research was to design and analyze the psychometric properties of the Inventory to Evaluate the Effectiveness of the Work Teams (IET). The following phases were carried out: development of the preliminary version of the inventory based on the IMOI model (Inputs-Mediators-Outputs-Inputs), validation of the test and analysis of its psychometric properties. The total sample consisted of 286 employees from organizations in Bogota city, 211 women (73\%) aged between 22 and 66 years old (M=33.1 and $\mathrm{SD}=9.9)$ and 75 men between 18 and 68 years old $(\mathrm{M}=32$ and $\mathrm{SD}=11.3)$. The final version of IET is constituted by 46 items for the measurement of three theoretical dimensions and distinguishes the following factors in each dimension: in the input dimension: leadership (7 items), teamwork (6 items) and, climate and organizational culture (5 items); in the mediators dimension: action and transition processes (6 items) and team climate (4 items) and, finally, in the group and organizational level of results (15 items), and awards and rewards ( 3 items). It is concluded that the IET has adequate reliability and evidence of content and construct validity.
\end{abstract}

Keywords: work teams effectiveness, work teams, IMOI model, effectiveness assessment, work teams effectiveness evaluation

\footnotetext{
${ }^{1}$ Psicóloga, Especialista en Administración: Gerencia de Recursos Humanos, Magister en Psicología Organizacional y del Trabajo, Doctoranda en Salud, Psicología y Psiquiatría. Profesora Asociada e Investigadora, Universidad El Bosque, Facultad de Psicología. Avenida Carrera 9a No. 131 A - 02. Bogotá, Colombia. Tel.: (57-1)6489030 - (57)3153399872. Correo: leopsicopos@gmail.com (correspondencia), delgadoaleonor@unbosque.edu.co

${ }^{2}$ Psicóloga. Universidad El Bosque, Facultad de Psicología. Avenida Carrera 9a No. 131 A - 02. Bogotá, Colombia. Tel.: (57-1) 6489030. Correo: vleonr@unbosque.edu.co

${ }^{3}$ Psicóloga. Universidad El Bosque, Facultad de Psicología. Avenida Carrera 9a No. 131 A - 02. Bogotá, Colombia. Tel.: (57-1) 6489030. Correo: apinzonr@unbosque.edu.co

${ }^{4}$ Psicólogo. Universidad El Bosque, Facultad de Psicología. Avenida Carrera 9a No. 131 A - 02. Bogotá, Colombia. Tel.: (57-1) 6489030. Correo: amarinc@ unbosque.edu.co
} 


\section{Introducción}

Los cambios a nivel económico y tecnológico de las últimas décadas han generado nuevas estrategias corporativas que requieren estructuras organizacionales articuladas por equipos, como unidades básicas de trabajo. Esto faculta a las organizaciones para hacer frente a las exigencias de flexibilidad (Delgado, Romero, \& Gómez, 2008) que les impone un entorno caracterizado por una economía global, cambiante y orientada al cliente (Mathieu, Gilson, \& Ruddy, 2006). Además, les permite ser más eficientes que aquellas con estructuras tradicionales centradas en el trabajo individual (Hoegl \& Gemuenden, 2001; De la Torre, 2011) para afrontar tareas complejas y de diversa naturaleza, a través de la integración y la motivación de sus miembros (Rico, SánchezManzanares, Gil, Alcover, \& Tabernero, 2011). En consecuencia, la efectividad y la comprensión de los desafíos que los sistemas multi-equipo plantean, constituyen factores clave para el éxito de las organizaciones contemporáneas.

En este estudio se adopta la definición de equipos de trabajo presentada por Kozlowski e Ilgen (2006) porque reúne varios elementos presentes en las perspectivas de otros investigadores en este campo (Hackman, 1987; Guzzo \& Dickson, 1996; Kozlowski \& Bell, 2003), a saber: "colectivos que existen para realizar tareas organizacionalmente relevantes, que mantienen un cierto grado de interdependencia tanto en términos de objetivos como de tarea, gestionan y mantienen sus límites y están inmersos en un contexto organizacional que limita su actividad e influye sobre sus intercambios con otros equipos dentro de la organización" (p. 79).

La definición del constructo efectividad de los equipos de trabajo ha representado cierta dificultad para los investigadores en este campo (El Ansari et al, 2016; Mahembe \& Engelbrecht, 2014; Delgado et al, 2008). En términos generales, la efectividad se ha definido como la capacidad de los equipos de trabajo para activar sus propios recursos en pro del logro de sus objetivos (Sjøvold, 2006; Jaca, Viles, Mateo, Santos, \& Tanco, 2013)

En la literatura sobre el tema se pueden diferenciar dos tipos de enfoques a la hora de definir la efectividad de los equipos. El primero es unidimensional y suele utilizar medidas objetivas $\mathrm{y}$ absolutas para determinar el rendimiento del equipo o bien el nivel de productividad real alcanzado; el segundo enfoque es multidimensional, considera la efectividad como algo más que el rendimiento o la productividad y obliga a contemplar diversas dimensiones $\mathrm{y}$ variables dentro de su espacio conceptual (Mahembe \& Engelbrecht, 2014).

En las últimas décadas se han desarrollado diferentes modelos teóricos que intentan describir, explicar y comprender los procesos y factores asociados a la efectividad de los equipos de trabajo, los cuales han evolucionado, incluido nuevos factores y ahondado en la relación entre ellos. Estos se han desarrollado bajo el esquema del modelo Input-Procesos-Output (IPO) de McGrath (1964, citado por Rico, Alcover de la Hera, \& Tabernero, 2010) y del modelo IMOI (Inputs-Mediadores-Outputs-Inputs) de Ilgen, Hollenbeck, Johnson, \& Jundt (2005).

Para el presente trabajo se tomó como punto de referencia el Modelo IMOI de Ilgen et al (2005). En este modelo, según Rico et al (2010), los insumos son del contexto organizacional, del equipo $\mathrm{y}$ de los integrantes. El nivel organizacional contempla los sistemas de dirección de recursos humanos, el diseño de la organización, el clima y la cultura organizacional. En el contexto del equipo se hace referencia a la autonomía, la interdependencia y el liderazgo. El contexto de los integrantes se analiza desde el punto de vista de tamaño, cambio y diversidad.

Los mediadores son mecanismos psicosociales que facilitan a los miembros del equipo combinar sus recursos para llevar a cabo la tarea asignada por la organización (Rico et al, 2010). Entre estos se encuentran diferentes factores que suponen acciones de los miembros, así como los estados afectivos, cognitivos o motivacionales (Jaca et.al., 2013; Viles, Jaca, Tanco, \& Medina, 2011). También se incluyen los procesos que aparecen durante el trabajo en equipo y que influyen en el desempeño del mismo (Viles, Zárraga-Rodríguez, \& Jaca, 2013).

Entre los mediadores se encuentran los procesos, los estados emergentes y mediadores mixtos (Viles et al, 2011). De acuerdo con Rico et al (2010), existen procesos de la tarea, interpersonales 


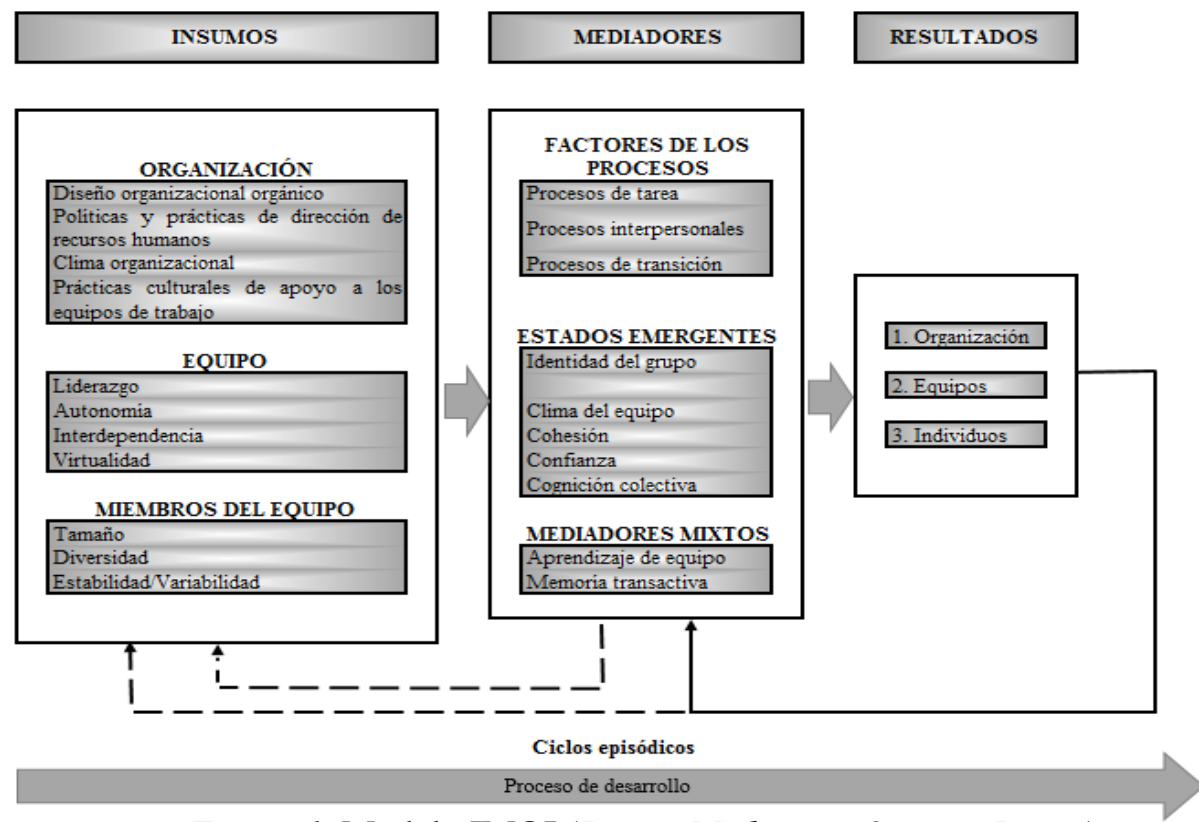

Figura 1. Modelo IMOI (Inputs-Mediators-Outputs-Inputs)

Fuente: elaboración propia, a partir de Rico et al (2010)

y de transición; en cuanto a los estados emergentes, se han estudiado principalmente los siguientes: potencia, eficacia de equipo, clima de equipo, cohesión, confianza y cognición compartida. Los mediadores mixtos no son fácilmente clasificables en las dos categorías anteriores, ya que hacen parte de una mezcla entre procesos y estados emergentes, son: el aprendizaje del equipo y los sistemas de memoria transactiva.

Con respecto a los resultados, Mathieu, Maynard, Rapp y Gilson (2008) enfatizan en una perspectiva de análisis multidimensional y multinivel. Por ello, Rico et al (2010) proponen tres niveles: organizacional, de equipo y de rol; y tres dimensiones: desempeños (las acciones relevantes para conseguir resultados), rendimiento (compuestos por las consecuencias del desempeño) y las actitudes. Teniendo en cuenta que es un proceso cíclico, los resultados se convertirán más adelante en nuevos insumos para el proceso de evaluación de la efectividad de los equipos (Viles et al, 2013).

Al considerar a los equipos de trabajo como sistemas multinivel (individual, grupal y organizacional) y su efectividad como un fenómeno emergente (Kozlowski \& Ilgen, 2006) resultante de patrones de interacción constante y frecuente entre sus miembros (Rico et al, 2010), la medición de tal efectividad debe ser coherente con esta conceptualización, pero hasta donde conocen los autores de este artículo los instrumentos de evaluación publicados no van en esta dirección.

En la literatura se reportan diversas herramientas para evaluar aspectos relacionados con los equipos de trabajo. Algunos se ubican en el nivel individual, por ejemplo, el cuestionario para medir comportamientos eficaces en los equipos de trabajo (Bonavia, Molina y Puchol, 2015) y el instrumento denominado evaluación comprehensiva de la efectividad de los miembros de equipos (Ohland et al, 2012).

Otros están orientados a evaluar la efectividad de los equipos de trabajo en contextos ocupacionales específicos: la escala de efectividad de equipos en comunidades de salud mental para adultos (El Ansari et al, 2016), la escala para evaluar el funcionamiento del equipo directivo de los centros educativos (Ahumada, Montecinos, \& Sisto, 2008) y la herramienta para evaluar el funcionamiento de los equipos de trabajo en entornos docentes (Viles et al, 2013).

Por otra parte, están los instrumentos que miden algunas dimensiones de efectividad, centrados en los equipos. Campion, Medsker y Higgs (1993) diseñaron un instrumento compuesto por 54 ítems que evalúa diseño del trabajo, interdependencia, composición del equipo, contexto y procesos. Fernández y Juárez (2001) evalúan dos dimensiones asociadas con la efectividad de los equipos de trabajo: contexto y procesos de equipo. Dentro de la dimensión de contexto se incluyen características del equipo, 
características de la tarea, toma de decisiones, formación y apoyo de la dirección. La dimensión de procesos de equipo evalúa cultura de equipo, interdependencia y comunicación/cooperación. Ficapal-Cusí, Boada-Grau, Torrent-Sellens y Vigil-Colet (2014) presentan la adaptación al español de la Escala para el funcionamiento interno de los equipos de trabajo (QFI-22), que mide cooperación, comunicación, apoyo psicológico, planeación y organización del trabajo, manejo de recursos y apoyo para la innovación.

Por último, se encuentran los desarrollos evaluativos basados en modelos IPO e IMO. García-Salmones (2008), en el marco de un modelo IPO y con el objetivo de poner a prueba un modelo para explicar la satisfacción laboral y la eficacia grupal en el sector industrial, creó un cuestionario que mide variables input personales (edad y nivel de estudios), grupales (tamaño del grupo y heterogeneidad grupal), sobre la tarea (tareas directas y de vigilancia, tareas cognitivas, autonomía y feedback) y los estresores de rol (ambigüedad de rol y conflicto de rol). En procesos grupales mide cohesión, normas y el manejo eficaz de los conflictos. Las variables output consideradas son la satisfacción laboral y la eficacia grupal.

Jaca (2011), con base en el modelo IMOI y desde la perspectiva de la sostenibilidad de los equipos de mejora, produjo dos herramientas distintas: el cuestionario de evaluación de entradas y resultados (IMO-IT Organización) y la autoevaluación de los equipos de mejora (IMO-IT Equipos). Por su parte, los trabajos de Viles et al (2011), Jaca et al (2012) y Viles et al (2013) aportan una propuesta metodológica de evaluación integral del modelo IMOI, basada principalmente en entrevistas, de los diferentes factores a nivel de insumos, mediadores $y$ resultados en organizaciones de los sectores automotriz, hospitalario, industrial y educativo.

Estos trabajos son los antecedentes más próximos al desarrollo del IET, por cuanto proponen estrategias evaluativas de la efectividad de los equipos de trabajo, desde la perspectiva del modelo IMOI completo; sin embargo, están focalizadas sobre los procesos de calidad en sectores económicos puntuales en España. En consecuencia, estas herramientas de evaluación podrían tener un sesgo natural hacia estos criterios de certificación de calidad y ver limitado su alcance cuando la evaluación de la efectividad de los equipos de trabajo se oriente a otros propósitos al interior de las organizaciones.

El objetivo de la presente investigación fue diseñar y analizar las propiedades psicométricas del Inventario para evaluar la efectividad de los equipos de trabajo - IET.

\section{Método}

Para este estudio se llevaron a cabo las siguientes fases: desarrollo de la versión preliminar del inventario, validación de la prueba y análisis de sus propiedades psicométricas.

En primer lugar, el desarrollo de la versión preliminar del inventario tuvo tres momentos: una revisión bibliográfica exhaustiva, la construcción de la estructura de la prueba con base en el Modelo IMOI y la elaboración de los ítems.

La revisión bibliográfica sobre efectividad de equipos de trabajo, en las principales bases de datos científicas para psicología y ciencias administrativas, partió de dos estudios anteriores (Delgado-Abella, Meneses, Carvajalino, \& Toro, 2015; Delgado-Abella, \& Gallardo, 2016). Esta revisión se realizó para indagar sobre referentes conceptuales y empíricos del tema objeto de estudio, hacer un inventario de los instrumentos de evaluación existentes y elegir el modelo teórico que fundamentara la prueba, ya que, en consonancia con lo planteado por Casullo, "el modelo sirve como marco de referencia para poder construir los datos" (2009, p. 17). La búsqueda se efectuó utilizando los siguientes descriptores, en inglés y en español: equipos de trabajo, efectividad de los equipos de trabajo, modelos de efectividad de los equipos de trabajo, evaluación de la efectividad de equipos de trabajo.

El paso anterior permitió establecer que el modelo IMOI es uno de los más completos y aceptados en el ámbito académico, como marco conceptual y teórico de alto valor para diferentes estudios (Viles et al, 2013; Delgado-Abella et al, 2015). Así entonces, se tomó como base para el diseño del inventario el planteamiento de Rico et al (2010) y para la construcción de la estructura de la prueba se procedió a la elaboración de una 
matriz conceptual con la definición de cada uno de los elementos del modelo IMOI, a partir de la literatura sobre equipos de trabajo.

El diseño de ítems para evaluar cada aspecto de la matriz conceptual, atendió a los criterios de efectividad de los equipos de trabajo expuestos de manera comprehensiva por Rico et al (2010) y otros autores que han investigado sobre aspectos particulares de los equipos de trabajo (Fernández \& Juárez, 2001; Berenguer, Cerver, Ramón, \& Torre, 2004; Malec, et al, 2007; Delgado et al, 2008; Yang \& Guy, 2011; Anglada-Monzón, Tejero-González, \& Ruíz-Barquín, 2012; Ohland, et al, 2012; Viles et al, 2013; Barsade \& O’Neil, 2014; Feistritzer \& Jones, 2014; Driedonks, Gevers, \& Van Weelw, 2014; Amaya, 2014; Bonavia, Molina, \& Puchol, 2015; DelgadoAbella et al, 2015; Delgado-Abella \& Gallardo, 2016), pero que también hacen aportes puntuales al tema de la efectividad.

De la misma manera, el diseño de los ítems también estuvo orientado por una perspectiva pragmática del tema en organizaciones contemporáneas, lo cual se logró a través de la aplicación de una entrevista semiestructurada sobre efectividad de los equipos de trabajo, a tres expertos y a los miembros de sus respectivos equipos, en tres organizaciones de la ciudad de Bogotá: una empresa de telecomunicaciones multinacional, una institución educativa y una organización no gubernamental (Delgado-Abella, Meneses, Montaña, \& Aguirre, 2014). Esto permitió tener en cuenta las características lingüísticas, culturales y situacionales de la población objetivo. Además, en concordancia con el proceder de Salessi y Omar (2016), se omitió deliberadamente el uso de antónimos y de oraciones negativas. A este punto, la primera versión del IET contaba con 145 ítems.

Como segunda fase de este estudio, para asegurar la validez de contenido de la prueba, los 145 ítems iniciales fueron evaluados por tres jueces expertos: una psicóloga organizacional con sólidos conocimientos en equipos de trabajo, un gerente de talento humano y un psicómetra experto en diseño de instrumentos de evaluación. Se les solicitó que evaluaran la pertinencia, escritura y lenguaje de cada ítem, en una escala de cuatro puntos (1. nada adecuado, 2. poco adecuado, 3. adecuado y 4. muy adecuado); además, se requirió que consignaran las razones de su calificación en el apartado de "observaciones" para cada ítem, dimensión y para toda la prueba. De igual manera, se les pidió que se pronunciaran sobre la suficiencia del conjunto de ítems para explorar la efectividad de los equipos de trabajo y cada una de sus dimensiones. Al final de esta etapa se realizaron los ajustes de acuerdo con las observaciones aportadas por los expertos, se eliminaron 15 ítems y la prueba quedó conformada por 130 ítems, después del proceso de validación de contenido.

La estructura de la versión preliminar del IET se ilustra en las Tablas 2, 3 y 4, allí se describe la matriz conceptual de la prueba y se definen sus tres dimensiones: insumos, mediadores $\mathrm{y}$ resultados, así como cada uno de sus componentes. Dentro de los insumos (Tabla 2), se hace referencia a cuatro aspectos: primero, la composición del equipo descrita en términos de la diversidad y el tiempo; segundo, tres características del liderazgo: compartido, externo y coaching; tercero, el diseño de la tarea y el contexto de trabajo de los equipos, desde el punto de vista de la autonomía y la interdependencia (de la tarea y los objetivos); y cuarto, en el contexto de la organización se consideran las prácticas culturales de apoyo a los equipos de trabajo, el diseño y el clima organizacional. La dimensión mediadores (Tabla 3) incluye procesos (acción, transición e interpersonales), estados emergentes (cohesión, identidad, potencia y eficacia, clima de equipo y confianza) y mediadores mixtos (aprendizaje de equipo y memoria transactiva). Por último, la dimensión resultados (Tabla 4) abarca tres niveles: primero, resultados individuales, desde el punto de vista afectivo y del desempeño del rol; segundo, del desempeño y viabilidad del equipo; y tercero, los resultados organizacionales. Para más información sobre cada uno de estos componentes, se sugiere revisar el artículo de Rico et al (2010).

En la tercera fase de este estudio, para obtener una estimación de la validez de Constructo del IET, de acuerdo con el modelo IMOI y su estructura conceptual, se ejecutó un Análisis Factorial Exploratorio (AFE) de cada una de las dimensiones propuestas: insumos, mediadores y resultados. También se llevó a cabo el 
procedimiento de obtención de indicadores de confiabilidad de la prueba.

\section{Participantes}

Se trabajó con una muestra por conveniencia, la cual estuvo compuesta por 286 empleados de varias organizaciones privadas de la ciudad de Bogotá, con una edad promedio de 31 años, el $36 \%$ fueron hombres y el $64 \%$ mujeres, distribuidos así: 211 mujeres $(73 \%)$ con edades comprendidas entre los 22 y los 66 años ( $\mathrm{M}=33.1$ y D.E.=9.9) y 75 hombres entre los 18 y los 68 años $(M=32$ y D.E.=11.3). Con respecto al nivel educativo, la distribución de la muestra fue la siguiente: bachilleres, 6\%; técnicos y tecnólogos, $28 \%$; profesionales, $41 \%$ y postgraduados, $25 \%$. Del sector salud provino el $75 \%$ de la muestra y el $25 \%$ restante, de organizaciones de otros renglones del sector servicios.

\section{Instrumento}

Después del proceso de validación de contenido, la versión preliminar del IET tenía 130 ítems en una escala de cinco puntos, así: (1) Totalmente en desacuerdo, (2) En desacuerdo, (3) De acuerdo, (4) Totalmente de acuerdo y (5) No sé o no aplica, esta última luego fue codificada como valor perdido. El protocolo de recolección de datos incluye un apartado diseñado para obtener información sobre características sociodemográficas de los empleados (edad, sexo, escolaridad, cargo y tipo de organización). Este instrumento se aplicó a la muestra.

En las Tablas 2, 3 y 4 se definen las dimensiones y subdimensiones de la matriz conceptual de la prueba, así como el número de ítems para cada una. Es importante enfatizar que lo que se pretende evaluar no son los constructos allí expuestos, como tal, sino su aporte o relación con la efectividad de los equipos de trabajo.

\section{Análisis de datos}

Se evaluó la pertinencia de la matriz de datos para realizar el análisis factorial exploratorio, se usó el coeficiente Kaiser-Meyer-Olkin y la prueba de esfericidad de Bartlett, para insumos (KMO=0.89 y Esfericidad de Bartlett $\chi 2=1315.78$, $g l=153, p=.000)$, para mediadores $(\mathrm{KMO}=0.89 \mathrm{y}$ Esfericidad de Bartlett $\chi 2=671.55, g l=45, p=.000$ ) y para resultados $(\mathrm{KMO}=0.93$ y Esfericidad de Bartlett $\chi 2=1493.77, g l=153, p=.000$ ), indicadores que evidencian la viabilidad de la muestra para llevar a cabo los análisis factoriales. Los datos se procesaron mediante SPSS versión 21.

\section{Resultados}

\section{Fiabilidad del IET}

Como un indicador de la medida de fiabilidad de la prueba, se usó el coeficiente Alfa de Cronbach. Todas las escalas del test mostraron valores adecuados de fiabilidad: en la dimensión insumos: liderazgo $\alpha=.91$, trabajo en equipo $\alpha=.83$ $\mathrm{y}$, clima y cultura organizacional $\alpha=.88$. En la dimensión mediadores: procesos de acción y transición $\alpha=.88$ y clima de equipo $\alpha=.84$. Por último, en la dimensión resultados las subescalas resultados a nivel grupal y organizacional $\alpha=.95$, y reconocimientos y recompensas $\alpha=.87$.

\section{Evidencias de validez de contenido y de constructo del IET}

Para obtener evidencia de la validez de contenido de la prueba, los 145 ítems de la versión inicial del test fueron valorados por tres jurados expertos bajo los criterios de pertinencia (P), escritura (E) y lenguaje (L) de cada ítem, en una escala de cuatro puntos (1. nada adecuado, 2. poco adecuado, 3. adecuado y 4. muy adecuado). El coeficiente de armonía interjueces Rwg (Finn, 1970) se usó como medida de correlación intraclase para datos categóricos basados en la varianza expresada por los jueces. Este coeficiente puede ser interpretado como indicador de validez de contenido y se basa en la proporción de la varianza observada sobre la esperada. Los resultados obtenidos para cada criterio y para el test completo se ilustran en la Tabla 1 y muestran niveles óptimos de acuerdo entre los tres jueces. Los coeficientes Rwg obtenidos para P, E, y L, se presentan en la Tabla 1.

Tabla 1. Coeficientes de armonía interjueces obtenidos en el desarrollo del IET

\begin{tabular}{lccc}
\hline Variable & $\begin{array}{c}\text { Rwg } \\
\text { M }\end{array}$ & $\begin{array}{c}\text { Rwg } \\
\text { DE }\end{array}$ & $\begin{array}{c}\text { Rwg } \\
\text { IC95\% }\end{array}$ \\
\hline Pertinencia & .92 & .19 & $.889-.951$ \\
Escritura & .88 & .26 & $.838-.922$ \\
Lenguaje & .90 & .25 & $.859-.941$ \\
Test total & .90 & .19 & $.869-.931$ \\
\hline Fuente: elaboración propia & &
\end{tabular}


Tabla 2. Estructura preliminar del IET - Dimensión Insumos: matriz conceptual y número de ítems

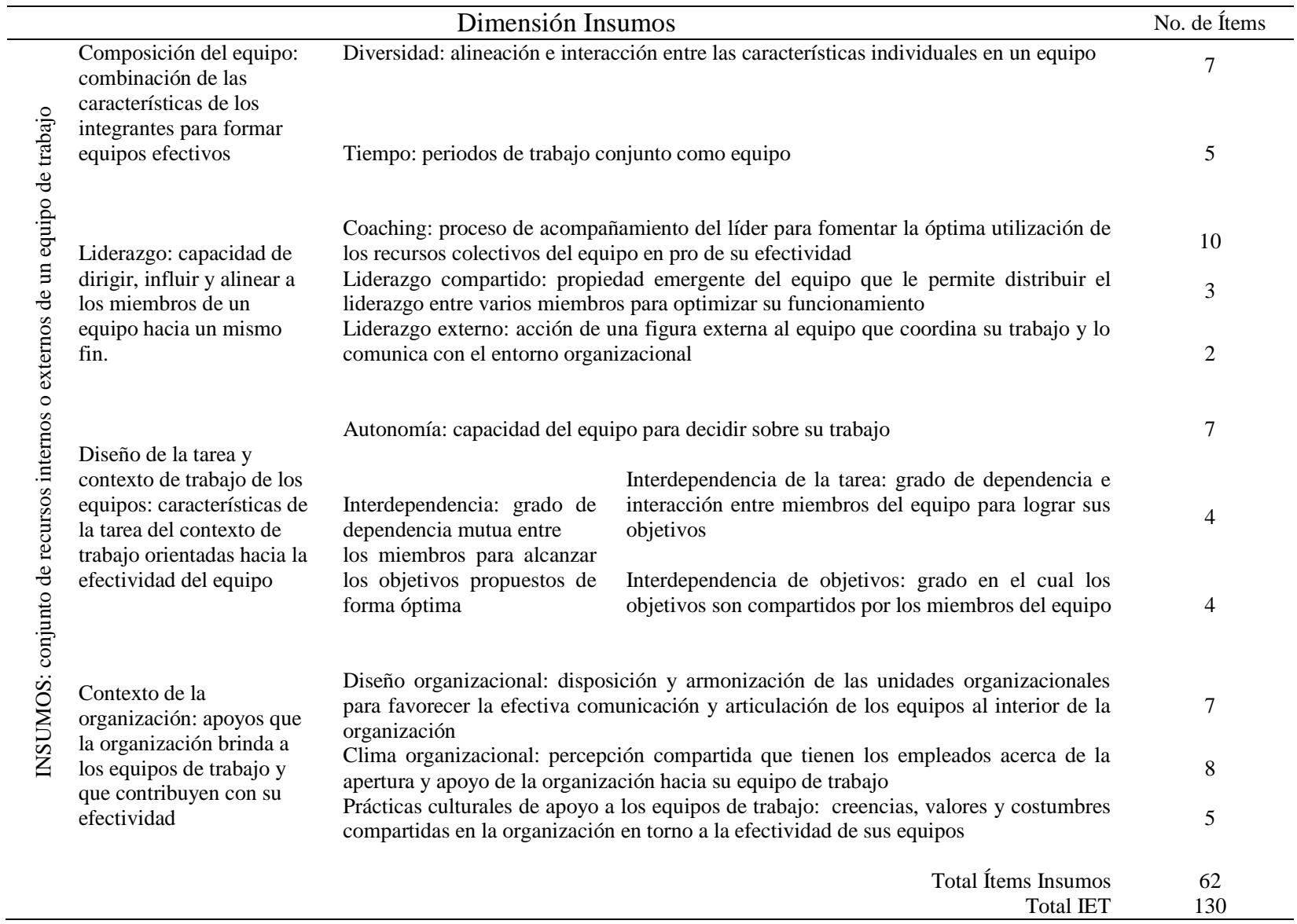

Fuente: elaboración propia a partir de Rico et al (2010)

Como se mencionó anteriormente, después de este proceso de validación de contenido se eliminaron 15 ítems y la prueba quedó conformada por 130 ítems, a partir de los cuales se realizaron los análisis factoriales que se presentan posteriormente.

De estos 130 ítems, la dimensión insumos aporta 62 ítems distribuidos en cuatro tipos de recursos internos que aportan a la efectividad de los equipos de trabajo, a saber: composición del equipo (12 ítems), liderazgo (15 ítems), diseño de la tarea y contexto de trabajo de los equipos (15 ítems), y contexto de la organización (20 ítems).

La dimensión mediadores contribuye con 48 ítems distribuidos así: procesos (25 ítems), estados emergentes (16 ítems) y mediadores mixtos (7 ítems).

En la última dimensión con 20 ítems, se muestran tres niveles: resultados individuales (4 ítems), resultados de equipos (11 ítems) y resultados organizacionales (5 ítems).

Para determinar la estructura interna del IET se ejecutó un Análisis Factorial Exploratorio (AFE), usando como método de extracción Mínimos
Cuadrados no Ponderados y Rotación Oblimin con normalización Kaiser, de cada una de las dimensiones propuestas: insumos, mediadores y resultados.

En la dimensión insumos, el AFE produjo la siguiente composición: el factor liderazgo agrupó los ítems i13, i14, i17, i19, i20, i21 e i22; el factor trabajo en equipo se conformó por los ítems i7, i8, i10, i35, i37 e i50; y el factor clima y cultura organizacional arrojó esta agrupación: i57, i59, i60, i61 e i62. Esta solución de tres factores explica el $68,47 \%$ de la varianza de la dimensión insumos. Para la dimensión mediadores, el AFE generó una solución de dos factores que explican el $65,72 \%$ de la varianza total, así: un primer factor denominado procesos de acción y transición incluyó los siguientes ítems: m6, m7, $\mathrm{m} 9, \mathrm{~m} 12, \mathrm{~m} 18$ y m20; y en el segundo factor se agruparon los siguientes ítems relativos al clima de equipo: $\mathrm{m} 22, \mathrm{~m} 23, \mathrm{~m} 39$ y m41. La solución de dos factores obtenida para la dimensión resultados, mediante el AFE, explica el 70,5\% de la varianza total; el primer factor reúne resultados a nivel grupal y organizacional, y contiene los 
Tabla 3. Estructura preliminar del IET - Dimensión Mediadores: matriz conceptual y número de Ítems

\begin{tabular}{|c|c|c|c|}
\hline & & Dimensión mediadores & No. de Ítems \\
\hline 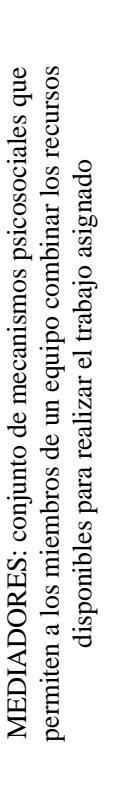 & $\begin{array}{l}\text { Estados Emergentes: estados } \\
\text { cognitivos, motivacionales o } \\
\text { afectivos del equipo, de } \\
\text { carácter dinámico y variable, } \\
\text { con respecto al contexto, } \\
\text { insumos, procesos y resultados } \\
\text { del equipo }\end{array}$ & $\begin{array}{l}\text { Cohesión: compromiso entre los integrantes del equipo y con su tarea } \\
\text { Identidad del equipo: grado en que una persona se define a sí misma como } \\
\text { integrante de un equipo } \\
\text { Potencia y eficacia de equipo: creencia compartida por los miembros de un } \\
\text { equipo sobre su capacidad para ser efectivos } \\
\text { Clima de equipo: percepción compartida por un equipo acerca de las } \\
\text { normas, actitudes y expectativas que operan en un contexto social } \\
\text { determinado } \\
\text { Confianza: disposición de una persona a ser influenciada por otra, con base } \\
\text { en las expectativas sobre la mutua relación al interior del equipo }\end{array}$ & $\begin{array}{c}14 \\
6\end{array}$ \\
\hline & & $\begin{array}{r}\text { Total Ítems Mediadores } \\
\text { Total IET }\end{array}$ & $\begin{array}{c}48 \\
130 \\
\end{array}$ \\
\hline
\end{tabular}

Fuente: elaboración propia a partir de Rico et al (2010)

Tabla 4. Estructura preliminar del IET - Dimensión Resultados: matriz conceptual y número de Ítems

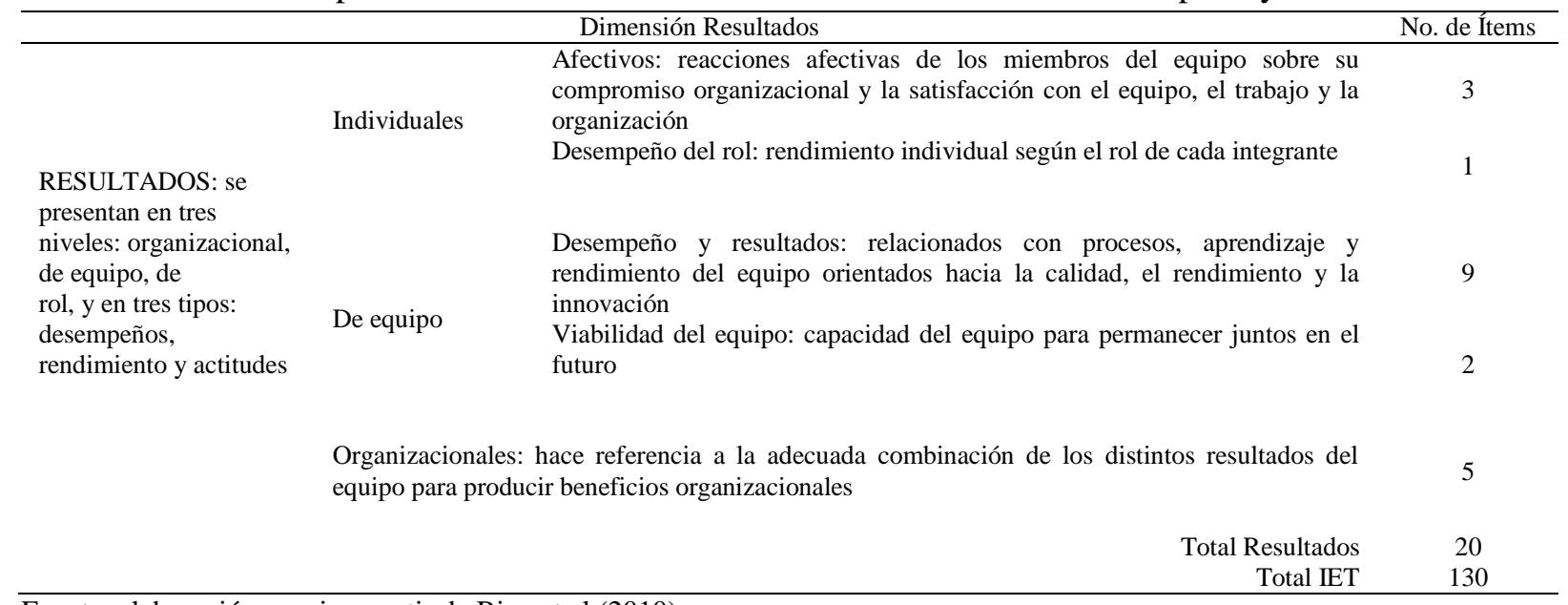

Fuente: elaboración propia a partir de Rico et al (2010)

siguientes ítems: r1, r2, r3, r8, r9, r10, r12, r13, $\mathrm{r} 14, \mathrm{r} 15, \mathrm{r} 16, \mathrm{r} 17, \mathrm{r} 19$ y r20; el segundo factor agrupa los ítems r4, r5 y r6, y hace referencia a reconocimientos y recompensas.

En resumen, el proceso de eliminación de ítems a partir de los AFE de cada una de las tres dimensiones del IET, produjo una reducción de más del 50\% en total. De 130 ítems quedaron 46 por las siguientes razones: se descartaron los ítems con cargas inferiores a .59 y aquellos que cargaban en varios factores.

Por último, en la Tabla 6 se presentan las correlaciones entre escalas y subescalas del IET, todas positivas y significativas $(p<.01)$. Se puede apreciar que, al interior de cada escala, las correlaciones entre subescalas oscilan entre $r=.47$ y $r=.57$, lo que implica que existe cierto solapamiento entre las dimensiones. Para las tres escalas, la correlación con sus respectivas subescalas es más alta (entre $r=.71$ y $r=.95$ ) y entre todas las subescalas de la prueba las correlaciones vuelven a ubicarse en un rango medio (desde $r h o=.39$ hasta $r h o=.72$ ). 
Tabla 5. Estructura factorial de cada una de las dimensiones del IET

\begin{tabular}{|c|c|c|c|c|c|c|c|c|c|}
\hline \multicolumn{4}{|c|}{ Insumos } & \multicolumn{3}{|c|}{ Mediadores } & \multicolumn{3}{|c|}{ Resultados } \\
\hline Ítem & $\begin{array}{l}\text { Trabajo } \\
\text { en } \\
\text { Equipo }\end{array}$ & $\begin{array}{l}\text { Lide } \\
\text { razgo }\end{array}$ & $\begin{array}{c}\text { Clima y } \\
\text { Cultura } \\
\text { Org. }\end{array}$ & Ítem & $\begin{array}{c}\text { Procesos } \\
\text { Acción y } \\
\text { Transic. }\end{array}$ & $\begin{array}{c}\text { Clima de } \\
\text { equipo }\end{array}$ & Ítem & $\begin{array}{l}\text { Resul } \\
\text { tados }\end{array}$ & $\begin{array}{l}\text { Recono } \\
\text { cimientos }\end{array}$ \\
\hline I7 & .592 & .474 & .314 & M6 & .799 & .512 & $\mathrm{R} 1$ & .646 & .307 \\
\hline I8 & .811 & .476 & .530 & M7 & .755 & .538 & $\mathrm{R} 2$ & .758 & .526 \\
\hline $\mathrm{I} 10$ & .719 & .487 & .439 & M9 & .688 & .428 & R3 & .671 & .521 \\
\hline I13 & .288 & .728 & .227 & M12 & .768 & .506 & R4 & .409 & .919 \\
\hline I14 & .405 & .851 & .400 & M18 & .676 & .401 & R5 & .392 & .867 \\
\hline $\mathrm{I} 17$ & .412 & .818 & .455 & M20 & .794 & .498 & R6 & .479 & .726 \\
\hline I19 & .524 & .681 & .469 & M22 & .429 & .721 & $\mathrm{R} 8$ & .796 & .319 \\
\hline $\mathrm{I} 20$ & .398 & .799 & .339 & M23 & .500 & .858 & R9 & .810 & .399 \\
\hline I 21 & .521 & .858 & .529 & M39 & .525 & .752 & $\mathrm{R} 10$ & .707 & .437 \\
\hline $\mathrm{I} 22$ & .424 & .801 & .495 & M41 & .564 & .728 & $\mathrm{R} 12$ & .677 & .394 \\
\hline I35 & .825 & .318 & .371 & & & & R13 & .693 & .481 \\
\hline I37 & .793 & .408 & .336 & & & & R14 & .768 & .410 \\
\hline I50 & .715 & .324 & .464 & & & & $\mathrm{R} 15$ & .707 & .500 \\
\hline I57 & .589 & .445 & .768 & & & & $\mathrm{R} 16$ & .775 & .257 \\
\hline I59 & .487 & .354 & .762 & & & & $\mathrm{R} 17$ & .851 & .225 \\
\hline I60 & .297 & .435 & .739 & & & & $\mathrm{R} 18$ & .850 & .211 \\
\hline I61 & .361 & .453 & .891 & & & & R19 & .874 & .287 \\
\hline I62 & .460 & .298 & .737 & & & & $\mathrm{R} 20$ & .758 & .407 \\
\hline
\end{tabular}

Tabla 6. Correlaciones entre escalas y subescalas del IET

\begin{tabular}{|c|c|c|c|c|c|c|c|c|c|}
\hline & 2 & 3 & 4 & 5 & 6 & 7 & 8 & 9 & 10 \\
\hline 1. Trabajo en equipo & $.52^{* *}$ & $.47^{* *}$ & $.80^{* *}$ & $.69^{* *}$ & $.68^{* *}$ & $.77^{* *}$ & $.66^{* *}$ & $.39^{* *}$ & $.66^{* *}$ \\
\hline 2. Liderazgo & - & $.55^{* *}$ & $.89^{* *}$ & $.63^{* *}$ & $.57^{* *}$ & $.67^{* *}$ & $.54^{* *}$ & $.40^{* *}$ & $.56^{* *}$ \\
\hline $\begin{array}{l}\text { 3. Clima y cultura } \\
\text { organizacional }\end{array}$ & & - & $.74^{* *}$ & $.55^{* *}$ & $.59^{* *}$ & $.61^{* *}$ & $.72^{* *}$ & $.61^{* *}$ & $.77^{* *}$ \\
\hline 4. Insumos & & & - & $.77^{* *}$ & $.73^{* *}$ & $.83^{* *}$ & $.71^{* *}$ & $.57^{* *}$ & $.73^{\text {** }}$ \\
\hline $\begin{array}{l}\text { 5. Procesos de acción } \\
\text { y transición }\end{array}$ & & & & - & $.57^{* *}$ & $.91^{* *}$ & $.65^{* *}$ & $.48^{* *}$ & $.69^{* *}$ \\
\hline 6. Clima de equipo & & & & & - & $.84^{* *}$ & $.69^{* *}$ & $.55^{* *}$ & $.69^{* *}$ \\
\hline 7. Mediadores & & & & & & - & $.76^{* *}$ & $.59^{* *}$ & $.79^{* *}$ \\
\hline $\begin{array}{l}\text { 8. Resultados grupal y } \\
\text { organizacional }\end{array}$ & & & & & & & - & $.52^{* *}$ & $.96^{* *}$ \\
\hline $\begin{array}{l}\text { 9. Reconocimientos y } \\
\text { recompensas }\end{array}$ & & & & & & & & - & $.71^{* *}$ \\
\hline 10. Resultados & & & & & & & & & - \\
\hline
\end{tabular}

**. $p<.01$ (bilateral)

En conclusión, el IET quedó conformado por 46 ítems, distribuidos así: tres dimensiones: insumos, mediadores y resultados, conformados por siete factores: en insumos, liderazgo ( 7 ítems), trabajo en equipo (6 ítems) y, clima y cultura organizacional (5 ítems); en mediadores, procesos de acción y transición (6 ítems) y clima de equipo (4 ítems) y, por último, resultados a nivel grupal y organizacional (15 ítems), y reconocimientos y recompensas (3 ítems).

\section{Discusión}

El interés por el estudio de los equipos de trabajo se ha incrementado en los últimos lustros 
debido a que el diseño de las organizaciones muestra una creciente tendencia a generar estructuras articuladas por equipos. La efectividad de tales equipos resulta de suma importancia para el óptimo desarrollo de las organizaciones.

El objetivo de este trabajo fue presentar el Inventario para Evaluar la Efectividad de los Equipos de Trabajo - IET y sus propiedades psicométricas, en el marco de los planteamientos del Modelo IMOI (Input-Mediator-Output-Input), desarrollado por Ilgen et al. (2005), revisado y actualizado posteriormente por Mathieu et al. (2008) y Mathieu, Gallagher, Domingo y Klock (2019), entre otros. Este modelo entiende la efectividad del equipo de trabajo como un concepto complejo y multidimensional que se analiza desde perspectivas diferentes y complementarias: insumos de la organización, el equipo y sus integrantes (Jaca, 2011), los mediadores y resultados; además, es uno de los más completos y aceptados en el ámbito académico, como marco conceptual de alto valor para diferentes estudios (Viles et al, 2013; Delgado-Abella et al, 2015).

El diseño de los ítems partió de la construcción de una matriz conceptual, fruto de una revisión bibliográfica sobre criterios de efectividad en cada uno de los elementos contemplados por el modelo IMOI, así como del modelo completo. De manera complementaria, se tuvo en cuenta la visión de expertos que están vinculados directamente con equipos de trabajo en las organizaciones. Por tanto, el IET se sustenta en la investigación académica existente y, a la vez, está permeado por una visión pragmática de la efectividad de los equipos de trabajo.

De acuerdo con la propuesta teórica del modelo IMOI, para estimar la validez de Constructo del IET se realizaron AFE de cada una de las dimensiones: insumos, mediadores $y$ resultados. Esto permitió depurar el inventario mediante la eliminación de ítems en cada facto siguiendo las recomendaciones de Lloret-Segura, Ferreres-Traver, Hernández-Baeza y TomásMarco (2014).

Con respecto a la dimensión insumos, el AFE produjo una composición de tres factores que explica el $68.47 \%$ de su varianza: liderazgo, clima y cultura de la organización, y trabajo en equipo. Tomando como referencia la matriz conceptual
(Tabla 2) elaborada a partir del modelo IMOI (Rico et al, 2010), es interesante apreciar que tanto el factor liderazgo como el factor clima y cultura organizacional conservaron los ítems pertenecientes a la subdimensión conceptual de su mismo nombre; mientras que el factor trabajo en equipo se compone de seis ítems que recorren el espectro de la dimensión insumos, excepto de la subdimensión liderazgo, incluyendo aspectos de la composición del equipo (diversidad y tiempo), diseño de la tarea y contexto de trabajo de los equipos (interdependencia de tarea) y del contexto de la organización (clima organizacional).

En el caso de la dimensión mediadores, la solución de dos factores obtenida explica el $65,72 \%$ de su varianza y genera una clara separación entre los procesos de acción y transición orientados hacia la tarea (Rico et al, 2010; Mathieu et al, 2019) y aspectos propios del clima de equipo en un marco de valores tales como la justicia, la comunicación, el respeto y la confianza. Sobre este segundo factor, clima de equipo, la literatura sobre estados emergentes reporta evidencia empírica que vincula la efectividad de los equipos de trabajo, respectivamente, con un clima de justicia (Cole, Carter y Zhang, 2013); un clima de "libertad de expresión" -group voice climate- (Morrison, Wheeler-Smith, \& Kamdar, 2011; Duan, Xu, \& Frazier, 2019); un clima de seguridad psicológica, que conlleva respeto (Mathieu et al, 2019); y por último, un clima de confianza (Brahm \& Kunze, 2012).

Para la dimensión resultados se encontró una estructura compuesta por dos factores claramente diferenciados que explican el $70.5 \%$ de su varianza. El primero de ellos agrupa ítems relativos a calidad, eficiencia y eficacia del trabajo del equipo y a la contribución estratégica del equipo a los resultados de la organización. El segundo factor reúne ítems que tienen que ver con recompensas y reconocimientos a nivel individual y grupal. Esta solución bifactorial de la dimensión resultados se alinea de cierta manera con la conceptualización de efectividad de equipos propuesta por Mathieu et al (2019), quienes establecen dos grandes categorías de resultados, a saber: unos tangibles, es decir, producto de la interacción del equipo (productividad, eficiencia y calidad); y otros, más centrados sobre los 
miembros del equipo, a nivel individual y colectivo. En esta última categoría se encuentran los reconocimientos y recompensas.

La composición de la estructura factorial del IET encontrada en esta investigación va en la dirección expuesta por Mathieu et al (2019) al mostrar cierto sano traslape entre diversas categorías conceptuales al interior del modelo IMOI, ya que, la investigación actual conduce a pensar que los insumos, los mediadores y las características estructurales de los equipos de trabajo se superponen, coevolucionan y combinan colectivamente de forma dinámica para generar efectividad (p.19). Se trata entonces de una perspectiva no lineal capaz de explicar y representar con mayor claridad un fenómeno tan complejo como la efectividad de los equipos de trabajo.

Los resultados obtenidos muestran que se ha creado un instrumento de evaluación de la efectividad de los equipos de trabajo con adecuada confiabilidad, evidencia de validez de contenido y de constructo. No obstante, por tratarse de un instrumento nuevo es necesario profundizar en la investigación sobre sus propiedades psicométricas para alcanzar un modelo de medida con óptimo ajuste, robusto y parsimonioso.

En la misma dirección, en futuras investigaciones con muestras de mayor tamaño, se podría indagar en qué medida se replica y enriquece la estructura factorial presentada en este artículo, ya que según Rico et al (2010), en la investigación sobre efectividad de los equipos de trabajo desde el modelo IMOI, se han encontrado problemas de validez discriminante en temas relacionados principalmente con aspectos afectivos. Adicionalmente, es importante continuar el estudio de otras propiedades psicométricas del IET, como validez convergente, discriminante y análisis de invarianza.

La estructura factorial del IET (Tabla 5) incluye un par de factores con pocos ítems cada uno, clima de equipos con cuatro y recompensas con tres, que si bien no resulta ideal tampoco es despreciable; ya que, según Lloret-Segura et al (2014, p.1157) corresponde a una condición moderada "cuando disponemos de comunalidades entre .40 y .70 , y el número de variables por factor es de 3-4 ítems", con un tamaño muestral de 200 casos.

Como fortalezas de este estudio se pueden mencionar las siguientes: se presenta a la comunidad académica una herramienta para evaluar la efectividad de los equipos de trabajo desde la perspectiva completa del modelo IMOI y abierto a todo tipo de organizaciones, es decir, su uso no se centra en un segmento ocupacional específico. Es el resultado de varios estudios previos a partir de revisiones bibliográficas exhaustivas y ha consultado también a expertos del sector real.

A partir de este inventario, y sus desarrollos posteriores, será posible desarrollar investigación relacionada con la efectividad de los equipos de trabajo para fortalecer el cuerpo teórico de este constructo tan importante para la psicología organizacional y el mundo de las organizaciones contemporáneas. El IET permitirá identificar aquellas variables posibles de intervenir y que pueden ser clave para contribuir al mejoramiento de la efectividad de los equipos de trabajo al interior de las organizaciones. Cabe anotar que, para evaluar un tema tan complejo como la efectividad de los equipos de trabajo, el abordaje debe ser a través de metodologías mixtas y utilizando diversas fuentes de indagación multinivel. Por otra parte, este inventario mide la efectividad desde la perspectiva de la percepción de los integrantes de los equipos; en consecuencia, se requiere que la medida de efectividad sea complementada desde el punto de vista de los directivos, clientes y otros. De igual forma, conviene anotar que la muestra estuvo conformada por empleados de organizaciones privadas de la ciudad de Bogotá, con un claro predominio del sector servicios y en especial del ámbito de la salud; esto lleva a pensar en la necesidad de probar la bondad psicométrica en otros fragmentos del mundo organizacional y del trabajo.

Las limitaciones principales estuvieron vinculadas a la consecución de la muestra, ya que el instrumento preliminar estaba compuesto por 130 ítems que implicaba la dedicación de aproximadamente una hora para diligenciarlo y eso dificultó un poco el acceso a los trabajadores. $\mathrm{Si}$ bien el test reducido permite disminuir el tiempo de aplicación, deben realizarse análisis 
complementarios para comprobar que las propiedades psicométricas se mantienen al administrar únicamente los ítems seleccionados, por otra parte, la correlación entre dimensiones sugiere la posibilidad de una estructura factorial jerárquica con factores de primer y segundo orden que debería ser evaluada mediante técnicas de análisis factorial confirmatorio.

Los lectores que se encuentren interesados en usar el IET para fines de investigación, pueden solicitarlo al correo electrónico de la primera autora.

\section{Referencias}

Ahumada, L., Montecinos, C., \& Sisto, V. (2008). Desarrollo y validación de una escala para evaluar el funcionamiento del equipo directivo de los Centros Educativos. Interamerican Journal of Psychology, 42(2), 228-235. Recuperado de http://pepsic.bvsalud.org/pdf/rip/v42n2/v42n2 a05.pdf

Anglada-Monzón, P., Tejero-González, C. M., \& Ruiz-Barquín, R. (2012). Programas de formación "Adventure Based Counseling". Un estudio sobre el desarrollo de competencias vinculadas al trabajo en equipo. Anales $D e$ Psicología, 28(3), 939-945. doi:10.6018/analesps.28.3.156181

Amaya Arias, A. (2014). Adaptación transcultural y validación del instrumento observacional de medición del trabajo en equipo para cirugía (Observational Teamwork Assessment for Surgery - OTAS) en el contexto colombiano (Tesis de maestría inédita). Universidad Nacional de Colombia, Bogotá, Colombia. Recuperado de http://www.bdigital.unal.edu.co/43125/1/3772 4987.2014.pdf

Barsade, S., \& O'Neill, O. (2014). Employee Teamwork Scale [Database record]. Retrieved from PsycTESTS. doi:10.1037/t37753-000

Berenguer, G., Cerver. E., Ramón, T., \& Torre, A. (2004). El estilo directivo de las mujeres y su influencia sobre la gestión del equipo de trabajo en las cooperativas valencianas. CIRIEC-España, Revista de Economía Pública, Social y Cooperativa, (50), 123-149.
Recuperado de http://www.redalyc.org/pdf/174/17405007.pdf Bonavia, T., Molina, J., \& Puchol, A. (2015). Validez estructural de un cuestionario para medir comportamientos eficaces en los equipos de trabajo. Anales de Psicología, 31 (2), 667-676. doi:10.6018/analesps.31.2.184041

Brahm, T., \& Kunze, F. (2012). The role of trust climate in virtual teams. Journal of Managerial Psychology, 27(6), 595-614. doi:10.1108/02683941211252446

Campion, M., Medsker, G., \& Higgs, A. (1993). Relations between work group characteristics and effectiveness: Implications for designing effective work groups. Personnel Psychology, 46(4), 823-847. doi:10.1111/j.1744-6570.1993.tb01571.x

Casullo, M. M. (2009). La evaluación psicológica: Modelos, técnicas y contextos. Revista Iberoamericana de Diagnóstico y Evaluación - e Avaliação Psicológica, 1(27), 9-28. Recuperado de http://www.aidep.org/sites/default/files/201707/R271.pdf

Cole, M. S., Carter, M. Z., \& Zhang, Z. (2013). Leader-team congruence in power distance values and team effectiveness: The mediating role of procedural justice climate. Journal of Applied Psychology, 98(6), 962. doi:10.1037/a0034269

De la Torre, J. (2011). La gestión de equipos: Heterogeneidad en la destreza, rendimiento de los recién llegados y toma de decisiones medioambientales (Tesis doctoral inédita). Universidad de Granada, Granada, España. Recuperada de http://digibug.ugr.es/bitstream/10481/15437/1 /19587880.pdf.

Delgado, M., Romero, A., \& Gómez, L. (2008). Teams in organizations: A review on team effectiveness. Team Performance Management: An International Journal, 14(1/2), 7-21. doi:10.1108/13527590810860177

Delgado-Abella, L., \& Gallardo, L. (2016). Diseño y validación de contenido de un instrumento que evalúa las condiciones individuales y grupales para la efectividad de los Equipos de Trabajo - IEET. (Tesis de maestría inédita). Universidad El Bosque, Bogotá, Colombia. 
Delgado-Abella, L., Meneses, A., Carvajalino, P., \& Toro, R. (2015). Diseño y validación de contenido de un instrumento que evalúa condiciones organizacionales para la efectividad de los equipos de trabajo (Tesis de maestría inédita). Universidad El Bosque, Bogotá, Colombia.

Driedonks, B., Gevers, J., \& Van Weele, A. (2014). Success factors for sourcing teams: How to foster sourcing team effectiveness. European Management Journal, 32(2), 288304. doi:10.1016/j.emj.2013.01.009

Duan, J., Xu, Y., \& Frazier, M. L. (2019). Voice climate, TMX, and task interdependence: A team-level study. Small Group Research, 50(2), 199-226. doi:10.1177/1046496418805855

El Ansari, W., Lyubovnikova, J., Middleton, H., Dawson, J., Naylor, P., \& West, M. (2016). Development and psychometric evaluation of a new team effectiveness scale for all types of community adult mental health teams: A mixed-methods approach. Health and Social Care in the Community, 24(3), 309-320.

doi:10.1111/hsc.12203

Feistritzer, N., \& Jones, P. (2014). A proof-ofconcept implementation of a unit-based advanced practice registered nurse (APRN) role: Structural empowerment, role clarity and team effectiveness. The Nursing Clinics of North America, 49(1), 1-13. doi:10.1016/j.cnur.2013.11.009

Fernández, N., \& Juárez, C. (2001). La eficacia de los equipos de trabajo y su medición. Boletín De Estudios Económicos, 56(172), 57-84. Recuperado de http://ezproxy.unbosque.edu.co:2048/login?ur l=https://search-proquest-

com.ezproxy.unbosque.edu.co/docview/13487 64554 ?accountid $=41311$

Ficapal-Cusí, P., Boada-Grau, J., Torrent-Sellens, J., \& Vigil-Colet, A. (2014). Spanish adaptation of the internal functioning of the work teams scale (QFI-22). Psicothema, 26(2), 273-278. doi:10.7334/psicothema2013.148

Finn, R. H. (1970). A note on estimating the reliability of categorical data. Educational and Psychological Measurement, 30(1), 71-76. doi:10.1177/001316447003000106

García-Salmones Fernández, L. (2008). Satisfacción y eficacia: Un estudio con grupos de trabajo en un entorno productivo (Tesis doctoral inédita). Universidad Nacional de Educacion a Distancia, España. Recuperada de http://espacio.uned.es/fez/eserv/tesisuned:PsicologiaLgarcia/Documento.pdf

Guzzo, R. A., \& Dickson, M. W. (1996). Teams in organizations: Recent research on performance and effectiveness. Annual Review of Psychology, 47(1), 307-338. doi:10.1146/annurev.psych.47.1.307.

Hackman, J. R. (1987). The design of work teams. En Lorsch, J. (Ed.), Handbook of Organizational Behavior (pp. 315-342). Englewood Cliffs: Prentice-Hall.

Hoegl, M., \& Gemuenden, H. (2001). Teamwork quality and the success of innovative projects: A theoretical concept and empirical evidence. Organization Science, 12(4), 435-449. doi:10.1287/orsc.12.4.435.10635

Ilgen, D. R., Hollenbeck, J. R., Johnson, M., \& Jundt, D. (2005). Teams in organizations: From input-process-output models to IMOI models. Annual Review of Psychology, 56, 517-543.

doi:10.1146/annurev.psych.56.091103.070250

Jaca, C., Viles, E., Tanco, M., Mateo, R., \& Santos, J. (2013). Teamwork effectiveness factors in healthcare and manufacturing industries. Team Performance Management: An International Journal, 19(3/4), 222-236. doi:10.1108/TPM-06-2012-0017

Jaca, C. (2011). Modelo de evaluación para la sostenibilidad de los equipos de mejora. (Tesis doctoral). Universidad de Navarra, Donostia, España. Recuperada de http://dadun.unav.edu/bitstream/10171/28160/ 1/Carmen_Jaca.pdf.

Kozlowsi, S. W., \& Ilgen, D. R. (2006). Enhancing the effectiveness of work groups and teams. Psychological Science in the Public Interest, 7(3), 77-124. doi:10.1111/j.1529-1006.2006.00030.x

Kozlowski, S. W., \& Bell, B. (2003). Work groups and teams in organizations. En Borman, W. C., Ilgen, D. R. \& Klimoski, R. J. (Eds.). Industrial and Organizational Psychology (pp. 333-375). Chichester: John Wiley \& Sons. 
Lloret-Segura, S., Ferreres-Traver, A., HernándezBaeza, A., \& Tomás-Marco, I. (2014). El análisis factorial exploratorio de los ítems: Una guía práctica, revisada y actualizada. Anales de Psicología, 30(3), 1151-1169. doi:10.6018/analesps.30.3.199361

Mahembe, B., \& Engelbrecht, A. (2014). The relationship between servant leadership, organisational citizenship behaviour and team effectiveness. Journal of Industrial Psychology, 40(1), 1-10. doi:10.4102/sajip.v40i1.1107

Malec, J. F., Torsher, L. C., Dunn, W. F., Wiegmann, D. A., Arnold, J. J., Brown, D. A., \& Phatak, V. (2007). The Mayo High Performance Teamwork Scale: Reliability and validity for evaluating key crew resource management skills. Simulation in Healthcare: The Journal of the Society for Simulation in Healthcare, 2(1), 4-10. doi:10.1097/SIH.0b013e31802b68ee

Mathieu, J. E., Gilson, L. L., \& Ruddy, T. M. (2006). Empowerment and team effectiveness: An empirical test of an integrated model. Journal of Applied Psychology, 91(1), 97-108. doi:10.1037/0021-9010.91.1.97.

Mathieu, J., Maynard, M. T., Rapp, T., \& Gilson, L. (2008). Team effectiveness 1997- 2007: A review of recent advancements and a glimpse into the future. Journal of Management, 34 (3), 410-476. doi:10.1177/0149206308316061

Mathieu, J. E., Gallagher, P. T., Domingo, M. A., \& Klock, E. A. (2019). Embracing complexity: Reviewing the past decade of team effectiveness research. Annual Review of Organizational Psychology and Organizational Behavior, 6, 17-46. doi:10.1146/annurev-orgpsych-012218-015106

Morrison, E. W., Wheeler-Smith, S. L., \& Kamdar, D. (2011). Speaking up in groups: A cross-level study of group voice climate and voice. Journal of Applied Psychology, 96(1), 183. doi:10.1037/a0020744

Ohland, M., Loughry, M., Woehr, D., Finelli, C., Bullard, L., Felder, R., Finelli, C., Layton, R., Pomeranz, H., \& Schmucker, D. (2012). The comprehensive assessment of team member effectiveness: Development of a behaviorally anchored rating scale for self and peer evaluation. Academy of Management Learning \& Education, 11(4), 609-630. doi: 10.5465/amle.2010.0177

Rico, R., Alcover de la Hera, C. M., \& Tabernero, C. (2010). Efectividad de los equipos de trabajo: Una revisión de la última década de investigación (1999-2009). Revista de Psicología del Trabajo y de las Organizaciones, 26(1), 47-71. doi:10.5093/tr2010v26n1a4

Rico, R., Sánchez-Manzanares, M., Gil, F., Alcover, C. Ma ${ }^{\mathrm{a}}$, \& Tabernero, C. (2011). Procesos de coordinación en equipos de trabajo. Papeles del Psicólogo, 32(1), 59-68. Recuperado de http://www.papelesdelpsicologo.es/pdf/1140.p df

Salessi, S., \& Omar, A. (2016). Desarrollo y validación de una Escala para Medir Actuación Emocional en el Trabajo. Revista Iberoamericana de Diagnóstico y Evaluacion - e Avaliação Psicológica, 1 (41), 66-79. Recuperado de http://www.aidep.org/sites/default/files/article s/R41/Art6.pdf

Sjøvold, E. (2006). Maturity and effectiveness in small groups. Nordic Psychology, 58(1), 4356. doi:10.1027/1901-2276.58.1.43

Viles Diez, E., Zárraga-Rodríguez, M., \& Jaca García, C. (2013). Herramienta para evaluar el funcionamiento de los equipos de trabajo en entornos docentes. Intangible capital, 9(1), 281-304. doi:10.3926/ic.399

Viles, E., Jaca, C., Tanco, M., \& Medina, A. (2011). Desarrollo metodológico para medir el trabajo en equipo. Revista Venezolana de Gerencia, 16 (55), 375-389. Recuperado de http://www.redalyc.org/articulo.oa?id=290205 61003

Yang, S., \& Guy, M. (2011). The effectiveness of self-managed work teams in government organizations. Journal of Business and Psychology, 26(4), 531-541. doi:10.1007/s10869-010-9205-2 